\title{
RABELAIS, MISE EN BOUCHE
}

\author{
Karine DOUPLITZKY \\ Berkeley University of California
}

\begin{abstract}
This paper examines Rabelais's many linguistic references to the mouth - both as an organ of taste and a tool of language. It also offers connexions between the grotesque body as described by Rabelais and the iconographic context of the French Renaissance that provides different representations of the mouth.
\end{abstract}

Keywords : Alcofribas; mouth; grotesque- tongue; body language; Erasmus; Bakhtin.

Mots-clés : Alcofribas; Bouche; corps grotesque; langue; language du corps; Erasme; Bakhtin.

\section{Introduction}

À chaque époque son regard sur la «bouche »: oracle, rictus du comédien, béance de l'enfer, gargouille, bouche-trou, bouche grotesque, fine bouche... j'aime à croire que cette bouche, qui s'inscrit comme une béance au milieu du visage, peut signifier différentes lectures du monde - si ce n'est bouleverser la face du monde.

Il s'agit ici d'ausculter la bouche rabelaisienne dans tous ses états - langue, palais, gosier, larynx, dents incluses - qui trouve son prolongement dans l'estomac et sa finitude là où la parole jaillit, comme une excrétion. Prendre Rabelais au pied de la lettre, c'est explorer la topographie de la bouche et entrer avec le narrateur dans l'espace buccal de son personnage principal, donc participer à une « mise en bouche ». ${ }^{1}$ ' 'est trouver refuge dans la langue, dans sa double acception d'organe et de principe d'oralité. L'organe se superpose à ses mises en mots.

J'entends ces jeux de mots comme une façon d'entrer en sympathie avec le texte, à partir de motifs relatifs à la culture française qui trouvent leur racine chez Rabelais comme chez les humanistes. Je suggère de m'appuyer sur une déconstruction du texte, à partir de ma position de lectrice moderne, pour mettre au jour une poétique, avec ses références au passé et son originalité historique. Jouant de la même technique que Rabelais, le faiseur de bons mots, l'inventeur de contrepèteries, canulars et galéjades, de jeux intertextuels, graphiques ou sonores, je me propose de faire résonner le texte - plus que de le raisonner - et de voir comment le langage cru du corps peut s'entrelacer avec un discours plus élevé.

La bouche qui parle est inséparable de la langue qui goûte, métaphoriquement et matériellement. La bouche qui a soif est celle avide de ces mots qui ont du goût, tel un bon vin. Pour Rabelais, le langage des appétits du corps, bas et grotesque, va de concert avec la noblesse de pensée. A partir des métaphores de la bouche, organe organisateur du corps grotesque - défini en ouvertures, orifices, protubérances et excroissances - j'envisage donc un dévoilement de cette parole festive qui s'inscrit dans les méandres du corps.

${ }^{1}$ François Rabelais, Les Cinq Livres, Jean Céard, Gérard Defaux, Michel Simonin eds., La Pochothèque (Paris, Le Livre de Poche, 1994), II.XXVIII, 519. 
En partant de la bouche bée comme posture rabelaisienne, qui trouve sa logique dans la définition de l'assoiffé - tels Pantagruel ou Gargantua qui ont soif de savoirs - je me propose de replacer le motif de la bouche dans le contexte iconographique de la Renaissance et de décrire son évolution depuis la bouche de l'enfer, dévorante, à celle grand ouverte sur la folie. L'éloge paradoxal de la folie, retournée en sagesse par Érasme, trouve un équivalent littéraire chez Rabelais qui s'appuie sur le topos du monde à l'envers pour accompagner son narrateur dans ses pérégrinations en terre buccale. La bouche, désignée comme vaste mise en abyme du récit, nous permettra de conclure sur sa fonction communicante, à l'image d'un corps grotesque ouvert à la réciprocité - ce qui s'entend, dans le contexte chrétien, comme une preuve de charité.

\section{Bouche bée}

Gargantua, à peine sorti du ventre de sa mère, crie : «à boire! à boire! à boire! ». ${ }^{2}$ Cri par trois fois répété, preuve qu'il n'est pas que cri, mais tout à la fois expression d'un besoin physiologique et d'un désir humain : la soif est aussi soif de paroles. L'enfant fait son entrée en société en ouvrant la bouche et en s'identifiant ainsi au groupe. Le «à boire » est une sorte de baptême linguistique, permettant de réitérer la consonne $« \mathrm{~b} »$, comme $« \mathrm{~b} »$ outeille, $\ll \mathrm{b} »$ ouche, « $B$ »adebec. Son père, Grandgousier, constate alors : «que grand tu as, supple le gousier. » D'où le nom de Gargantua qui lui est attribué, paraphrasant celui de son père et partageant les mêmes consonances gutturales. L'image de la bouche comme organe (incluant gorge et gosier) fait naître le récit. C'est l'image nourricière par excellence. Elle s'accompagne du parti pris linguistique pour l'oralité qui habite l'ensemble des Cinq livres. Le rapport de Pantagruel à la bouche est tout aussi explicite, par le truchement de la soif, comme le suggère l'auteur :

« Car Panta en Grec vaut autant à dire comme tout, et Gruel en langue Hagarène vaut autant comme altéré : voulant inférer, que à l'heure de sa nativité le monde était tout altéré. Et voyant en esprit de prophétie qu'il serait quelque jour dominateur des altérés. » ${ }^{3}$

L'étymologie fantaisiste que propose Rabelais rattache non seulement Pantagruel au monde des "altérés", autrement dit des assoiffés, mais elle lui confère aussi une connotation christique. Il compare la venue au monde de Pantagruel, né un vendredi, alors que la terre sue et qu'apparaissent signes et prodiges, à celle du messie (celui qui sauvera son peuple). Pantagruel, lui-même assoiffé, sauvera donc son peuple de la soif. Plus tard dans le récit, l'armée des Pantagruelistes portera en ses armoiries la devise : «champ de gueulle». Pantagruel est doublement né de la bouche populaire, tout d'abord par sa longue filiation gargantuesque telle qu'Alcofribas (anagramme de l'auteur), en tant qu'historiographe, nous la raconte. ${ }^{4}$ Il descend droit de Hurtaly (le sauvé des eaux) « beau mangeur de souppes » (fig. 1), d'Eryx «inventeur du jeu des gobelets », puis d'Etion qui est mort de la vérole pour avoir dormi «la gueule baye», etc.

\footnotetext{
${ }^{2}$ Rabelais, I.VI, 41.

${ }^{3}$ Rabelais, II.II, 311

${ }^{4}$ Rabelais, II.I, 299.
} 
S'en suivent d'autres noms de géants, grands masticateurs, pour lesquels il faut ouvrir bien grand la bouche afin de prononcer correctement leur nom.

Cette manière grossière d'introduire des personnages, dénués de bonnes manières, trouve un équivalent visuel dans la tradition des scènes de genre qui se développent au nord de l'Italie à la fin du XVI ${ }^{\mathrm{e}}$ siècle, avec l'école Bolognaise. Ainsi l'image du mangeur de soupe (fig. 1), représenté par Annibal Carrache désacralise le corps et illustre les mauvaises manières populaires dans un contexte profane. Le personnage, pris sur le vif, la bouche ouverte, est pour l'époque une manière de caractériser un type social, sans idéalisation, une façon également de sous-entendre un message moral en représentant son antithèse. Rabelais est passé maître dans ce type d'éloge paradoxal, décrivant des appétits monstrueux et une sensibilité boulimique pour prôner, dans la dialectique du récit, un esprit de mesure et de commensalité.

Pantagruel appartient à la tradition populaire également par l'emprunt de son nom à un petit diable du Mystère des Actes des Apôtres de Simon Greban. ${ }^{5} \mathrm{Ce}$ petit diable de la mer vient la nuit jeter du sel dans la gorge des ivrognes! De même Pantagruel est celui qui, plutôt que de désaltérer, donne soif, soif de paroles, soif de la parole divine. Sa fonction est «altérative». Ce nouveau messie ouvre les bouches et fait pendre les langues. Par ses références répétées à l'altération, Rabelais suggère l'image d'un «peuple élu », bouche ouverte et langue pendante «tirant la langue comme lévriers qui ont couru six heures »- et qui, nourri de ce sel, se montre toujours plus assoiffé de savoirs. ${ }^{6}$ Doit-on penser la soif comme préalable à toute connaissance, divine ou profane ? Non seulement Pantagruel donne soif, mais il est lui-même assoiffé ; c'est là une condition à sa future appartenance au peuple qu'il dominera, les Almyrodes (signifiant d'ailleurs en Grec saumâtre, salé). Tel le Christ qui s'est fait homme pour sauver les hommes, Pantagruel est né assoiffé, pour sauver les assoiffés.

Le sel occupe une place prépondérante dans le récit, comme élément nourricier. La contiguité du mot sel avec le mot sapere - «savoir», mais aussi «qui a du goût»- ouvre cet élément/aliment sur de multiples perspectives interprétatives. Il permet d'assainir l'eau, conserve les viandes et préserve les corps de la putréfaction; cela est à l'origine de sa valeur rituelle et marchande. ${ }^{7}$ Il est gage de vie, puisqu'il incite à boire et donne goût aux aliments, ainsi que du piquant à l'existence (donc au récit). S'il fait office de monnaie dans les civilisations primitives, il prend une connotation fiscale dès le Moyen Age avec le célèbre impôt sur le sel, la Gabelle, monopole royal et cause de nombreux soulèvements populaires (les plus importants en 1542 et 1548). Faut-il rappeler que la vallée de la Loire - pays de Rabelais - est sur cette grande ligne de commerce du sel qui va des grands marais salants de l'Atlantique, vers le centre du pays ? L'Anjou est précisément le pays des faux-sauniers et contrebandiers du sel.

\footnotetext{
${ }^{5}$ Rabelais, note $18,310$.

${ }^{6}$ Rabelais, II.II, 309.

7 Selon le médecin Paracelse, savant-aventurier et alchimiste dont les travaux ont marqué le XVIe siècle, le sel est l'un des trois «principes hypostatiques », avec le mercure et le soufre, qui constitue tout corps. Cette substance idéale se rattache à la terre en tant qu'elle est solide et stable ; elle possède des vertus solvantes et coagulantes qui déterminent consistance et saveur. Le sel est donc le corps de l'homme ; son principe salé demeure comme matière cristalline indestructible.
} 
La guerre de Pantagruel contre les Almyrodes en est un plaisant écho. Dans la Bible, le sel prend une tournure métaphorique: «Vous êtes le sel de la terre. Mais si le sel vient à s'affadir, avec quoi le salera-t-on? » (Mt 5,13). Le sel est un signe ; « le sel de la terre » évoque le disciple, lui-même signe de la présence du Christ. Son originalité est toutefois de nourrir par le manque : toute substance salée suscite d'abord une demande. C'est la réponse à un besoin corporel, celui d'étancher sa soif et d'avaler un liquide - on pense au vin. Par déduction, cela devient une quête spirituelle, à laquelle le vin répond à nouveau par sa référence chrétienne au sang du Christ. La soif a, de plus, une connotation sexuelle chez Rabelais de par la salivation qui l'accompagne: il en est ainsi, par exemple, quand la haute dame de Paris, dont Panurge est tombé amoureux, hésite à céder à ses avances (ou plutôt à ses promesses de «trésors », selon le proverbe: "la bourse ouvre la bouche ») : « ...par la vertu desquelles paroles, il lui faisait venir l'eau à la bouche $\gg^{8}$ L'image de l'eau à la bouche est à mettre en regard avec celle de l'eau bénite dont Panurge a voulu l'asperger quelques instants auparavant ! La référence à l'eau bénite est d'ailleurs explicite lors de la naissance de Pantagruel, utilisée à contre-emploi, dans un sens matériel et non spirituel:

«Encore, quand quelqu'un entrait en l'église, vous en eussiez vu à vingtaines de pauvres altérés qui venaient au derrière de celui qui la distribuait à quelqu'un, la gueule ouverte pour en avoir quelque gouttelette, comme le mauvais Riche, afin que rien ne se perdît. » ${ }^{9}$

A tous les niveaux de lecture donc, revient la figure de l'altération (au double sens de «soif » et de «modification»), directement corrélée à celle de la « gueulle ouverte »- posture rabelaisienne qui sert d'emblème à la dynastie gargantuesque. Ganrgantua a l'habitude de justifier son ingurgitation de vin au réveil en invoquant ses besoins physiologiques: «de ma nature je dors sallé. ${ }^{10}$ Et bien sûr, les cinq Livres de Rabelais se déroulent sous l'enseigne du vin, de ses bienfaits corporels et moraux. Le vin n'est pas seulement destiné à se soûler, mais à communier et à entrer en relation avec les dieux. Il va jusqu'à donner un sens au récit, à la recherche de la «Dive bouteille». L'image de la boisson se propage dans le texte par capillarité, jusqu'au lecteur. Au prologue du Tiers livre, Rabelais harangue ainsi son public :

«Bonnes gens, Beuveurs tresillustres, et vous Goutteux tresprecieux, veistez vous oncques Diogenes, le philosophe cynic?»

C'est à la posture du buveur que Rabelais en appelle ; traduit en termes corporels, cela évoque la bouche ouverte de l'ivrogne, buvant ou chantant, échappant une fois encore aux convenances. Le vin n'est pas que christique, il appartient au monde de Diogène, le philosophe dont la sagesse dérisoire, risible en apparence, gagne à être découverte en profondeur - si l'on en croit l'adage des « Silènes d'Alcibiades ». ${ }^{11}$ Humanisme et facétie se rencontrent dans les jeux de la

\footnotetext{
${ }^{8}$ Rabelais, II.XIX, 449.

${ }^{9}$ Rabelais, II.II, 309.

${ }^{10}$ Rabelais, I.XX, 111.

${ }^{11}$ Desiderius Erasmus and William Barker, The adages of Erasmus, Toronto, University of Toronto Press, 2001, p. 242.
} 
langue. Cette bouche, assoiffée et gloutonne, ouverte sur les plaisirs du corps s'oppose directement à « la petite bouche » de celui qui fait fi de la bonne chère. Cette opposition donnera naissance, dès le $\mathrm{XV}^{\mathrm{e}}$ siècle, à l'expression « faire la fine bouche ». En attendant, le vin qui désaltère est, chez Rabelais, joyeux et festif. Il renouvelle la sémantique visuelle, définissant la bouche bée dans le contexte de la soif, mais aussi du rire gras.

\section{Béance de l'enfer}

Cette image littéraire de la bouche ouverte croise celle de la tradition iconographique du $\mathrm{XVI}^{\mathrm{e}}$ siècle, essentielle pour mieux cerner les contours de la bouche grotesque rabelaisienne. Parions sur le fait que la richesse du contexte iconographique puisse efficacement démasquer ce que le texte de Rabelais, avec ses tournures parodiques et jeux de langage, masque des appétits pour la raison ou la religion. Remontons jusqu'à l'Antiquité pour rappeler que la bouche ouverte fait initialement référence à l'oralité : l'acteur déclamant ou l'oracle prophétisant (fig. 2). Mais cette bouche se lit en creux, béance pour le sculpteur, trou noir de la représentation et absence de matière. L'oralité ne se met pas en images: la peinture de la Renaissance en fera l'expérience, car les bouches restent obstinément fermées sur le message divin. C'est la gestuelle qui prend en charge le message oral. Même l'ange de l'annonciation n'ouvre pas la bouche: il se contente d'un geste de la main, parfois accompagné de phylactères, pour annoncer à Marie la nouvelle de sa fécondation. La bouche ouverte investira donc, à l'âge baroque, un terrain autre que celui de l'oralité - celui de la grimace et du masque social comme évoqué avec le mangeur de soupe, reformulation du masque antique de la comédie ou de la tragédie. Mais entre-temps, la béance concentre toutes les peurs et angoisse d'un monde en plein bouleversement. Au Moyen Age, la bouche ouverte est celle de l'enfer, ainsi représentée sur le manuscrit de Clèves (fig. 3). Selon l'Ancien Testament, les impies qui ont oublié Dieu descendent au shéol :

«C'est pourquoi le shéol dilate sa gorge et bée d'une gueule démesurée. Ils y descendent, ses nobles, ses foules et ses criards, et ils y exultent. » (Isaïe, 5:14)

Réplique de Saturne dévorant ses enfants (que Goya immortalisera magistralement), le diable, hideux, comme un lion rugissant, dévore les damnés (fig. 4). L'imaginaire de l'enfer est associé au tourment des sens : vacarme des démons et hurlements des suppliciés, odeur de putréfaction exhalée par les cadavres, fournaise des corps entassés dans leur prison étroite, soif ardente et faim dévorante du damné, torture par le feu rougi qui brûle jusqu'aux entrailles. Ces représentations frappantes des grandes peurs médiévales, que Jérôme Bosch traduira par un foisonnement d'images insolites (fig. 5), trouvent un équivalent littéraire chez Rabelais, dans l'infinie variation de ses figures linguistiques. Parallèlement, les spectacles populaires de l'époque, diableries ou mystères, traduisent sur scène ce répertoire de monstruosités et catalogue de tortures. En polarisant toute l'attention du public sur la gueule béante de l'enfer, cette scène exhibant corps brûlés dans le feu, mutilés ou écartelés, participe au développement d'un vocabulaire du corps grotesque dans lequel Rabelais puisera largement. Toutefois l'humanisation de la figure du Diable est en cours. 
Dans les marges des manuscrits médiévaux ou des monuments gothiques, comme l'a montré Michael Camille, se développe un univers interprétatif plus subtil, en même temps que subversif. ${ }^{12}$ L'ambivalence de la bouche, à la fois siège de la parole et de la mastication, est largement illustrée dans les marges par des personnages fantastiques tels que les sirènes, dragons, poissons, mi-hommes mianimaux... avalant des plantes, des animaux et en particuliers des lettres (fig. 6). Dans cette 'lettrophagie', par exemple, se nourrir de la parole de Dieu, plutôt que de la chair des animaux, est représenté «à la lettre ». Il s'agit d'une digestion ou rumination du parfum du texte. L'idée de 'saveur des écrits' prolonge une métaphore déjà utilisée chez Saint Augustin, qui renforce l'analogie entre lire et manger. ${ }^{13}$ Ce rapprochement fait d'autant plus sens dans la salle de réfectoire où les moines, rassemblés en communauté, lisent à voix haute la parole de Dieu tout en mangeant. Le banquet (de Platon à Rabelais) illustre de façon séculaire cette nécessité de satisfaire à la fois les appétits du corps et de l'esprit : savoir est, buvant \& mangeant.

Des exemples semblables d'art marginal peuplent les hauteurs des cathédrales. Invisibles du bas, des personnages grossiers, grimaçant, tirant la langue, moquent l'édifice en même temps que ses autorités (fig. 7). ${ }^{14}$ Ces créatures humaines et déformées sont logées entre les anges et les démons, comme un clin d'œil profane dans l'enceinte du sacré. Les gargouilles qui cernent les cathédrales gothiques fournissent un exemple similaire d'apprivoisement du diable et des monstres transformés en figures comiques. Ce sont des monstres réduits à leur bouche, pure corporalité, dont leur fonction inversée d'éjection (et non d'ingestion) est particulièrement stimulante pour penser Rabelais (fig. 8). D'ailleurs le mot gargouille partage la même étymologie que Grandgousier ou Gargantua et provient de goule (gorge). Les orifices y fonctionnent aussi à double sens: absorption de nourriture, comme défécation. Certains manuscrits médiévaux ne manquent pas de signaler la symétrie des deux orifices et représentent sans affectation des excréments devenus matière de dévotion (fig. 9). De façon similaire, prise de vin et pissement sont deux activités corporelles qui 'coulent' de concert chez Rabelais : "Venez vous icy culletans mon vin et compissez mon tonneau » (prologue du Tiers Livre). Pisser son malheur est aussi vital que boire : la pisse de Pantagruel, au chapitre XXIX, non seulement le guérit, mais forme des torrents qui serviront à guérir les autres. La pisse des géants crée des inondations, ce qui n'est pas sans rappeler la fonction des gargouilles comme pseudo-fontaines qui vomissent les pêchés du monde hors de l'enceinte sacrée de l'église. Contremodèle du corps du divin, elles servent de repoussoir autant que d'espace de liberté formel aux artisans sculpteurs. Avec pertinence, Michael Camille montre, à l'instar de celle de Mikhail Bakhtin dans le registre social, qu'à travers la dialectique des marges et du centre, le rire et la peur engagent un dialogue fécond,

\footnotetext{
${ }^{12}$ Michael Camille, Image on the edge : the margins of medieval art, Essays in art and culture, Cambridge, Mass., Harvard University Press, 1992.

${ }^{13}$ Camille, 64.

${ }^{14}$ Camille, 84.
} 
qui permet d'inclure des formes de dérision au sein d'un pouvoir autoritaire. ${ }^{15}$ Toutefois les marges, espace de liberté, restent au service dudit pouvoir, cantonnant dans une zone liminale la possible subversion. Malgré leur caractère transgressif, elles fonctionnent comme garantes de l'ordre établi. En cela Bakhtin suggère que les débordements du carnaval servent de soupapes pour négocier les tensions entre l'élite et le peuple, mais maintiennent finalement les différentiations culturelles entre hautes et basses classes sociales. C'est dans cet espace de négociation que le grotesque trouve sa naissance, la bouche du diable fusionnant avec celle du masque comique. Il y a donc convergence de ces deux paradigmes de la bouche, le masque et l'enfer, en une version grotesque, dont la hiérarchisation sociale, à la fin du XVI ${ }^{\mathrm{e}}$ siècle, donnera une version plus politique.

\section{Bouche folle}

Changement d'épistémè: l'Age Moderne canalise les grandes peurs du Moyen Age, auxquelles il substitue le concept de folie. Il tient désormais une position centrale dans la culture de l'Europe du Nord et hante l'imaginaire littéraire, ainsi que la culture visuelle. ${ }^{16}$ Sous l'influence des Humanistes, l'idée de folie s'infiltre à tous les niveaux de la société, de l'élite aux classes populaires et trouve un terrain commun d'inspiration. ${ }^{17}$ Elle permet de masquer l'anxiété d'une époque et de la refléter. Cette image de la folie, qui dénonce la vanité des appétits du monde, renouvelle l'iconographie de la Renaissance. Dès la fin du $X^{\mathrm{e}}$ siècle, la figure stéréotypée de l'insensé, telle que représentée dans les différentes versions de la Nef des fols de Brant (reprise plus tard chez Bosch (fig. 10) ou chez Bruegel), exprimait déjà la dérision et la déraison du monde. Avec Erasme, le concept se retourne : la sagesse de ce monde est folie et ce que le monde tient pour folie est sagesse. Erasme, pour bâtir son concept du fol-sage, s'appuie sur le paradoxe du christianisme, où le crétin - mot dérivé de chrétien à partir du trait sémantique d'innocence - est l'innocent sanctifié par l'église : bienheureux le pauvre d'esprit ! Le Christ, qui se prétendait roi des Juifs, n'avait-il pas été lui même qualifié de fou ? Et Salomon, le juste entre les justes, ne se présentait-il pas de même comme le plus fou des hommes ? Paul, dans l'Epître aux Corinthiens, avait déjà formulé cette idée d'interdépendance de la folie et de la sagesse : "Que celui d'entre vous qui paraît sage devienne fou pour être sage, car la folie de Dieu est plus sage que les hommes. »

Le fol, au sens d'Erasme, n'est donc pas le fou, contraire du sage, mais son envers. «Si tous les hommes sont fous, le seul homme sensé ne peut être que le fou véritable. ${ }^{18}$ Tel est le paradoxe sur lequel Rabelais va lui-même construire Pantagruel, fol parmi les fous. Le fol s'impose donc, dans son innocence et son extravagance, comme une figure positive de la Renaissance. Il est source de joie et d'énergie. Sage quand il rit, le fou enseigne la sagesse en nous faisant rire. Mais il

\footnotetext{
${ }^{15}$ Mihail Mihailovič Bahtin and Andrée Robel, L' Euvre de François Rabelais et la culture populaire au Moyen âge et sous la Renaissance Traduit du russe par André Robel ([Paris: Gallimard, 1970).

${ }^{16}$ Michel Foucault, Histoire de la folie à l'âge classique, Collection Tel (Paris: Gallimard, 1992).

${ }^{17}$ Yona Pinson, The fools' journey : a myth of obsession in northern Renaissance art (Turnhout: Brepols, 2008), 2.

${ }^{18}$ Maurice Lever, Le sceptre et la marotte histoire des fous de cour (Paris: Fayard, 2000), 183.
} 
a besoin du rire pour énoncer sa vérité. Rire, sagesse et folie trouvent d'ailleurs chez Rabelais une résonnance toute particulière, dans une boulimie lexicale qui utilise l'éloquence pour se moquer d'elle-même. La folie est follement réflexive.

Cette parole innocente et irresponsable du fou justifie sa présence auprès des souverains, qui considèrent que la vérité trouve refuge seulement chez les fous. Si les rois portent le sceptre, l'attribut du fou est sa marotte, un bâton surmonté d'une tête de fou et emblème dérisoire de son pouvoir. Cette marotte est le double du fou, lui-même étant le double ridicule du roi. La marotte est mise en abyme de la folie : un fou du fou. Elle est parfois remplacée par le miroir dans l'iconographie traditionnelle (fig. 11). Car le comble de la folie est que le fou, obsédé par son apparence dans le miroir, ne reconnaisse le reflet de son propre visage, ni ne reconnaisse sa folie. L'idée que le fou puisse être double, réfléchi par sa marotte, s'impose comme cliché visuel. Holbein l'a ainsi représenté en marge d'un de ses exemplaires de l'Eloge de la Folie d'Érasme (fig. 12).

Le fou, porte-parole de son époque et reflet superposable du roi, "personnage régnant», est représenté la bouche ouverte (fig. 11). Sorti des marges où il avait été confiné au Moyen-Age, il occupe désormais une position centrale dans la société, sur scène et dans la "page ». Ainsi la bouche dévorante des enfers s'estelle transformée en une bouche bée innocente. La bouche du fou libère la parole et devient emblématique d'une recherche de vérité.

Cette nouvelle interprétation iconographique de la bouche, telle qu'elle émerge à la Renaissance, offre un nouvel éclairage sur la bouche grotesque de Pantagruel. De la même façon que la vérité sort de la bouche des enfants et des ivrognes, elle sort de la bouche de ce grand fou de Pantagruel - un fou parfait qui montre la vanité de la sagesse et nécessité de la folie. ${ }^{19}$ Il exprime sa folle sagesse dans l'abondance du festif, énoncée comme une profession de foi : «un vray Cornucopie de joyeuseté et raillerie.»(prologue). Mais le fou, chez Rabelais, apparaît scindé en deux, comme le suggère Kaiser. ${ }^{20}$ Panurge est un fou, irrévérent, hors la loi, amuseur, libertin, perspicace et double de Pantagruel. Mais il apprend peu de ses expériences et bénéficie moins de la sagesse de sa folie. Pantagruel, au contraire, porte en lui l'essence véritable du fol-sage, ainsi qu'une forme de folie christique. Le bouffon qui divertit et le fol-sage qui philosophe forment, dès lors, un duo inséparable. La figure du fou se comprend comme un dialogue à partir de deux postures complémentaires, l'une fondée sur l'expérience, l'instinct et les forces naturelles (Panurge), l'autre sur la digestion de cette expérience ou raisonnement (Pantagruel). Cette même distinction qu'Erasme établit entre sentir le monde et le comprendre, Panurge et Pantagruel l'incarnent. Les fous vont parmi les sages mais les trouvent plus fous qu'eux et en reviennent. Eventuellement, comme Pantagruel l'a compris, il limite sa prise de parole et se contente de conclure, tandis que Panurge est tout langage. Il n'a pas de programme, il se nourrit des seules facéties du jour.

Reconnaître sa propre folie et lui donner la parole devient chez Erasme un précepte philosophique qui équivaut au « connais-toi toi-même» socratique. C'est,

\footnotetext{
${ }^{19}$ Walter Jacob Kaiser, Praisers of folly: Erasmus, Rabelais, Shakespeare, Harvard studies in comparative literature, (Cambridge,: Harvard University Press, 1963), 179.

${ }^{20}$ Kaiser, 127.
} 
du même coup, faire tomber les masques des faux-sages, ce à quoi Rabelais s'attèle par sa façon de mettre en dérision la langue artificielle des savants. L'éloge paradoxal du fou est un moyen d'enseigner la vérité par des moyens comiques, aboutissant à un comique de langage. La Renaissance porte le fou sur le devant de la scène littéraire et le texte se comprend comme un jeu spéculaire.

La bouche du fou, qui nous sert à définir la bouche grotesque, se double dès le XVe siècle d'un autre motif, celle du monde à l'envers. Quand le miroir dans lequel le fou se reflète se tourne vers le public, toute la société peut s'y refléter à son tour. C'est la signification du concept de Mundus stultus - le monde est fou, ou «le nombre des fous est infini », selon la formule de Salomon (fig. 13). La représentation du globe terrestre paré du capuchon du bouffon, orné d'oreilles d'âne et garni de grelots transcrit visuellement ce concept de folie universelle, qui ostensiblement reflète le vrai visage de la réalité. Le fou n'est plus en marge de la société, mais occupe bien son centre. Personne ne peut y échapper. Tel le bouffon de scène, le monde-fou, tout en conversant avec sa marotte, se tourne vers le public et pointe du doigt les inscriptions moralisantes énonçant les défauts de l'humanité. C'est bien de cette humanité entière qu'il rit : " vanité des vanités, tout est vanité !» (Ecclésiastes 1.2) Le topos du monde à l'envers, image clé d'un monde gouverné par la folie humaine, prône l'inversion des valeurs qui, quoique comiques, trahissent un sentiment général d'anxiété : les attributs royaux sont renversés, le haut prend la place du bas et le bouffon devient le roi du monde à l'envers! Rabelais construit la joyeuseté apparente de son récit sur cette anxiété véhiculée par l'image insolite d'un monde à l'envers - véritable charivari. L'entrée en bouche du narrateur coïncide avec l'image de cette bouche ronde et béante comme un globe terrestre, qui ouvre sur une nouvelle géographie.

\section{Mise en bouche}

Rabelais utilise cet imaginaire de la bouche, signe d'un monde à l'envers, pour explorer joyeusement et littéralement l'anatomie de la bouche, comme un récit dans le récit. La topographie de la bouche grotesque, telle qu'elle est établie au chapitre XXVIII de Pantagruel, tisse un entrelacs de significations qui opèrent tant sur le plan linguistique que visuel. Bakhtin a présenté comme une constante de la Renaissance ce phénomène d'assimilation des éléments cosmiques (tel le globe terrestre) dans les éléments du corps grotesque (ici la bouche) :

«...les gens assimilaient et sentaient en eux-mêmes le cosmos matériel avec ses éléments naturels dans des actes et fonctions du corps éminemment matériels : nourriture, excréments, actes sexuels (...) Le cataclysme cosmique, dépeint à l'aide d'images du bas matériel et corporel, est rabaissé, humanisé et transformé en un joyeux épouvantail. Ainsi le rire a vaincu la peur cosmique. " 21

Bakhtin interprète l'utilisation métaphorique de la bouche de Pantagruel dans le contexte de ce mouvement vers le «bas matériel » qui permet d'exorciser les folies du monde. Nous montrerons que les parties du corps grotesque sont disséminées dans l'écriture et que l'écrivain fait du verbe son propre corps.

${ }^{21}$ Bakhtin, 334. 
Rabelais n'est pas l'inventeur de l'exploration buccale. Comme l'a montré Auerbach, il s'est inspiré, pour ce chapitre, de deux sources, pour mieux s'en départir par la suite. ${ }^{22}$ La première source est populaire : il s'agit d'un passage des Grandes Chroniques de Gargantua, paru à Lyon en 1532, au cours desquelles une armée d'hommes pénétrait dans la bouche du géant pendant son sommeil pour l'étrangler. Cela convoque également les contes folkloriques où l'ogre avale les petits enfants et éventuellement les recrache. La seconde source est savante : il s'agit de l'Histoire Vraie de Lucien, ouvrage dans lequel un monde inconnu se révèle dans la "gueulle d'un cétacé », et où un naufragé se trouve amené à planter des choux. Le naufrage dans le ventre d'une baleine est une image qui hante d'ailleurs la littérature, depuis la Bible et Jonas, jusqu' au conte de Pinocchio ou au récit de Moby Dick. En 1556, Bruegel, reprenant une invention de Bosch, transcrit littéralement le proverbe à caractère moral : «Les gros poissons mangent les petits » en montrant l'image d'un cétacé anthropomorphe à l'immense bouche, avalant un poisson plus petit (fig. 14). Il traduit la folie des comportements humains par des jeux de disproportions et d'hybridation qui nous semblent entrer en résonance avec les jeux d'aberrations corporelles de Rabelais.

La «mise en bouche»d'Alcofribas dans Pantagruel, au chapitre XXVIII (Pantagruel), fonctionne comme une mise en abyme du récit. Elle suppose une mise en attente du récit fictionnel (attente qui va durer six mois, jusqu'à la sortie du narrateur de la bouche) et annonce un changement de locution: le héros, Pantagruel, se tait pour laisser la parole au narrateur, Alcofribas. Or une « mise en bouche », dans le langage culinaire, est aussi une mise en attente : c'est une sorte d'apéritif qui précède le repas et met en appétit. Elle a pour but de «mettre l'eau à la bouche ». La mise en bouche est avant-goût et promesse de quelque chose à venir. L'attente de pouvoir «par curieuse leçon, \& meditation frequente, rompre l'os, \& sucer la substantificque mouelle ${ }^{23}$ L'organe du goût appelle le goût du Verbe. Comme la soif, une «mise en bouche » prépare spirituellement le palais à un met délicieux. Cette attente ménage un suspense et tient en haleine. C'est la posture du pèlerin devant l'inconnu dont la curiosité est aiguisée : il se prépare à voyager vers une terre inconnue. La suspension du temps est présentée comme condition de découverte d'une nouvelle géographie et d'une nouvelle temporalité. Elle s'accompagne d'une suspension du langage chez Pantagruel - mais nous reviendrons sur ce point avec Erasme.

Ce temps préparatoire est utilisé par Alcofribas, (pseudo double de Rabelais) pour se présenter (« je, qui vous fais ces tant véritables contes»). Délogé de sa position confortable du « déjà-vu », il s'apprête à affronter une réalité inédite : le monde à l'envers dans la bouche du héros. Le voici, en attendant, abrité sous la langue de Pantagruel, comme sous un parapluie. ${ }^{24}$ Une langue qui couvre « comme une géline fait ses poulets », autrement dit une langue maternelle et nourricière qui protège. Que signifie «Parler sous le couvert de quelqu'un », si ce n'est adopter sa langue comme faisant nouvelle autorité ? Alcofribas « entre en langue » comme on

\footnotetext{
22 Erich Auerbach, Mimesis; the representation of reality in Western literature (Princeton,: Princeton University Press, 1953), 266.

${ }^{23}$ Rabelais, prologue à Gargantua, 9.

${ }^{24}$ Rabelais, II.XXVIII, 519.
} 
entre en religion. Il abandonne le mode fictionnel pour chercher celui, plus direct, qui colle à la «langue naturelle». Une image triviale se superpose à cette métaphore linguistique, celle du géant tirant la langue à demi, tel un fou qui échappe aux règles de la bienséance. Car seuls les fous, les gens du peuple ou les chiens tirent la langue. Tout mouvement de la bouche est sévèrement réprimé par les règles de bienséance. Rire fort, bâiller, cracher, roter, gonfler les joues, éternuer, se pourlécher, faire la moue, etc., enfreignent les codes comportementaux. On retrouve une fois de plus le grossier, source de la conception du grotesque. Les grossièretés, jurons et imprécations se répètent chez Rabelais comme éléments de description du «bas matériel». Pantagruel, en ouvrant la bouche et tirant la langue, participe à installer son image de fou, alors même que la folie est proposée comme programme spirituel.

Puis Alcofribas s'embarque pour un pèlerinage en terre folle. Il s'aventure sur la langue de Pantagruel, pont-levis vers l'imaginaire du héros:

«Donc, le mieux que je pus, montai par dessus, et cheminai bien deux lieues sur sa langue, tant que je entrai dedans sa bouche. $»^{25}$

Littéralement, il faut entendre que Pantagruel a l'auteur sur le bout de la langue. "Avoir quelque chose sur le bout de la langue", voici encore une référence à une parole qui ne sort pas, une parole retenue, un temps différé du récit, qui appelle à sa digestion. Silence inhabituel toutefois dans le récit rabelaisien où les langues sont généralement déliées par l'absorption de vin. Ici il y a interruption du bagout. L'idée qu'une temporisation est indispensable à la réflexion, est défendue par Erasme dans La Langue. Il prône une langue maitrisée et une parole opportune, où les émotions sont jugulées par la raison, ce qui permet de ne pas perdre le contrôle de son discours. Ce silence est donc d'abord à entendre, chez Erasmus, comme un bon goût : « se taire à propos ». Second éloge, le silence a pour vertu de faire exister les gestes pour libérer le sens. Car le mot est trompeur, tandis que le geste colle à sa désignation. Les actes peuvent donc être plus efficaces que la parole et le silence, devenir producteur de sens. Agir est présenté comme un remède à la parole. Cette vertu du silence est directement illustrée par la scène où Panurge, le clown qui amuse par ses pantomimes, développe une argumentation par signes devant Thaumaste. ${ }^{26} \mathrm{~L}$ 'image visuelle est substituée à la forme du discours scholastique. Les deux personnages gesticulent et croient se comprendre. Mais la gestuelle obscène de Panurge n'a de signifié que pour lui-même ; elle prend ironiquement un sens différent pour Thaumaste qui l'interprète de façon sérieuse et selon sa propre logique. La vérité d'un discours n'est que celle qu'on lui attribue et n'a pas valeur d'universel. Au cours de cette disputatio, les gestes de communication ont créé une tension sémiotique, donc une ambiguité. Le langage est brouillé par la multitude de signes, comme le suggère Jeanneret. ${ }^{27}$ Enfin le silence a pour troisième vertu de déclencher l'acte de parole, mais une parole naturelle, purifiée par l'attente. Le silence devient une force agissante qui annonce la pensée. Le fou qui amuse, tel Panurge, ne sait pas garder

\footnotetext{
${ }^{25}$ Rabelais, II.XXVIII, 519.

${ }^{26}$ Rabelais, II.XVIII, 433.

${ }^{27}$ Michel Jeanneret, Le défi des signes Rabelais et la crise de l'interprétation à la Renaissance, Collection L'atelier de la Renaissance (Orléans: Paradigme, 1994).
} 
le silence, sa parole est boniment. Au contraire, le langage est un supplément intempestif qu'il convient de maîtriser: ce rôle revient au fol-sage Pantagruel. D'où le proverbe répandu à l'époque : «Bouches en cœur aux sages, et cœur en bouche aux fols $\gg{ }^{28}$ Il y a toutefois un point d'anxiété lisible chez Erasme qui se demande si la parole n'échappe pas au sens. Mais Rabelais transforme cette angoisse en effet comique. La vérité toujours se cherche derrière l'invention des multiples langages, quoique arbitraires et affaires de convention; seule la langue naturelle ressurgie comme potentiellement authentique ; la langue que Panurge fait cracher à «l'escholier Limousin ».

À ce moment d'entrée en terre buccale pour Alcofribas, Pantagruel s'arrête soudain de forger des mots et tire la langue au langage. Redevenue simple organe de goût, la langue perd sa fonction de verbalisation et ne fait office que de vulgaire parapluie. Pourtant c'est au moment où elle se tait qu'elle fait le plus parler d'elle!

\section{5. Épopée en terre buccale}

Changement de décor, d'horizon et de fuseau horaire : la bouche se présente comme un seuil et une zone liminale d'inversion. Changement d'échelle qui caractérise le grotesque : le microcospique narrateur entre dans le macrocosme du géant. Comme le souligne François Rigolot (faut-il s'appeler ainsi pour devenir expert en rabelaiseries ?), la bouche du géant est le siège d'un lyrisme grotesque où « le cadre gigantal n'est là que pour servir de tremplin au gigantisme verbal ». ${ }^{29}$ Aussi la bouche grotesque est-elle l'occasion d'un foisonnement de nouveaux répertoires de modes d'expression. De la même manière qu'Erasme commençait sa réflexion sur la langue par une description détaillée de l'organe («charnue, parsemée de petites membranes et veinules, dodue, mais légère malgré tout et capable de se tourner facilement dans tous les sens »), Rabelais utilise la trivialité de la bouche comme jeu de langue, occasion d'une pluralité de sollicitations sémantiques. ${ }^{30}$ Le changement de perspective du narrateur sur son personnage implique un changement d'élocution : Alcofribas s'apprête à parler dans, et par, la bouche de sa créature et devient le porte-parole d'un récit documentaire. Il quitte l'intrigue «gigantale » pour revêtir la veste de l'explorateur. Car Pantagruel, incapable de raconter lui-même ce qui se trouve dans sa bouche merveilleuse, a besoin de ce relais pour que le récit continue. Alcofribas, "porte-parole», «porte» désormais une parole de vérité, liée à sa qualité de témoin et de découvreur d'un nouveau monde. Hélas, à peine a-t-il franchi le seuil buccal, qu'Alcofribas est parodié en sa qualité d'observateur du réel, par une formule emphatique et toute faite qu'il emploie, propre à une rhétorique éculée : «Mais ô dieux et déesses, que vis-je là ? » Dès que Rabelais intervient au sujet de la langue, c'est pour mieux se jouer d'elle, comme s'il voulait mettre en question l'instrument de son propre discours. Le projet d'authenticité de la parole, annoncé par un changement de registre du locuteur qui chemine sur la langue «naturelle » de son héros, est dès son origine bafoué par un reflux de formules de langage anciennes. Le projet de découverte d'une nouvelle nature est brouillé par un

\footnotetext{
${ }^{28}$ L'expression « cœur en bouche » signifie parler haut et fort.

${ }^{29}$ François Rigolot, Les langages de Rabelais, Études rabelaisiennes (Genève: Droz, 1972), 34.

${ }^{30}$ Erasme, La Langue, vol. 44, Histoire et Société (Labor et Fides, 2002), 83.
} 
langage qui ne peut se dépouiller de ses références anciennes. Une déception similaire attend le lecteur à qui «l'eau est monté à la bouche » à l'idée de découvrir un monde inconnu. Or la topologie supposée inédite de la bouche est ramenée à une géographie connue de l'ancien monde : les dents sont comme «les monts des Danois", la cavité buccale se ramène à Sainte Sophie de Constantinople, les villes ressemblent à Lyon ou Poitiers. Il y a, d'entrée en bouche, une difficulté à renouveler le langage puisqu'il s'appuie sur un système analogique usité. Constat identique quand le dialogue avec «l'étranger» (le planteur de choux) s'installe. Nouvelle déception. La réponse de l'étranger ne fait que paraphraser le visuel: «Je plante (dit-il) des choux. » L'inconnu repose sur le connu, et Rabelais se joue des complicités de l'écriture pour brouiller les pistes de l'interprétation. Son livre est fertile en attrapes de ce genre - attrape pigeons, attrape lecteurs. Que doit-on penser de cet autre étranger, ce «compagnon qui tendait au pigeon », rencontré juste après le planteur de choux ? Le pigeon bien parisien est la version basse de la colombe du Saint-Esprit et ce vol de pigeons de «l'autre monde », tel que le précise le compagnon, pourrait bien être porteur d'une annonciation :

«Lors je pensai que quand Pantagruel bâillait, les pigeons à pleines volées entraient dedans sa gorge, pensant que fût un colombier. ${ }^{31}$

L'attitude inconvenante du bailleur - réprouvée au nom de la bienséance (fig. 15) - est ironiquement sanctifiée, preuve d'un monde qui tourne à l'envers.

L'emphase d'Alcofribas, «ébahi » par tout ce qu'il voit, est employée bien mal à propos puisque dans bouche qu'il explore n'apparaît que le reflet du monde extérieur ordinaire : ce «nouveau monde » ou «terre neuve » (en allusion à la Terre-Neuve découverte par Jacques Cartier) ressemble étrangement à la Touraine natale de Rabelais : du cultivateur de choux, symbole du terroir (et on pense depuis à la comptine enfantine : «Savez-vous planter les choux?, à la mode, à la mode, savez-vous planter les choux ?, à la mode de chez nous... »), au braconnier, aux portiers et autres sénateurs rencontrés ainsi qu'aux maisons et villes, aux champs et forêts, à l'économie de marché, jusqu'à la maladie (en référence à la peste qui ravagea la Touraine en 1532 et1533) dont on meurt. Pourtant, cette réalité banale de la vie quotidienne, sur fond de bouche gigantesque, apparaît avec une certaine poésie, parce que le reflet qu'offre la bouche est fidèle, mais inversé : l'intérieur buccal est un extérieur ; le microcosme devient macrocosme ; l'exotique est familier ; le nouveau reflète l'ancien ; enfin l'argent est distribué à celui qui dort plutôt qu'à celui qui travaille. Une correspondance étroite est soulignée entre la physionomie externe du paysage et l'anatomie intérieure de la paroi buccale, avec ses hauteurs (dents) et dépressions, trous et abîmes. La surface buccale est bâtie selon l'image du corps grotesque, hybride, morcelé, parfois dépecé à la mode des descriptions anatomiques des romans de chevalerie. De nouvelles voix montent toutefois, participant de l'inversion : des personnages insignifiants, tel, précisément, le planteur de choux, qui d'ordinaire passeraient inaperçus, prennent soudain la parole et deviennent témoins oculaires, ce qui leur confère une incontestable prestance. Quand le géant se tait, c'est la rumeur populaire intérieure qui monte.

${ }^{31}$ Rabelais, II. XXVIII, 521. 
La bouche fonctionne comme un miroir et caisse de résonance du monde. ${ }^{32}$ Elle est interface qui sépare un imaginaire vraisemblable d'un réel invraisemblable. Allusion à la caverne de Platon, toutefois dépouillée de l'idéalisme d'un monde vrai des Idées, elle présente au contraire une vérité chancelante, morcelée en une multitude de points de vue, rappelant la parcellisation du corps joyeux et mouvant du carnaval. Par exemple, chacun juge le monde où il vit comme le plus ancien, l'autre étant le nouveau. Mais dès qu'on franchit le miroir, les notions de nouveau et d'ancien se brouillent. La relativité des perspectives de chacun sur le monde a raison de la vérité universelle. Le relativisme de Rabelais reflète ces moments troublés de la découverte du Nouveau Monde où les récits de voyage affluent et les voix se superposent.

La poursuite de l'expédition dans le gosier, jusqu'à l'estomac dans le chapitre suivant, s'accompagne d'un nouveau jaillissement d'images et métaphores de la digestion. Car les profondeurs corporelles sont fertiles, expression de puissance sexuelle, fécondité et abondance. Gosier est une ville qu'Alcofribas trouve « belle, bien forte, et en bel air », peuplée de Chrétiens (soulignant encore la tentation de vouloir retrouver, en terre étrangère, ce qui est familier). La qualité de l'air revient comme une préoccupation centrale d'Alcofribas, sans doute parce qu'à l'époque, les vents dans le corps trouvent leur explication théorique dans le recueil d'Hippocrate (contemporain de Rabelais), intitulé De flatibus, traitant « de l'air comme agent du monde ». Cette théorie de l'air est considérée avec d'autant plus de sérieux que la médecine est au centre des sciences (Rabelais étant lui-même médecin) et s'identifie avec la philosophie. Le corps est étudié comme échange, notamment par les courants d'air qui le traversent. Inhalations purifiantes et exhalaisons nocives. Flux d'air et reflux des pestifères, décrits comme une « infecte exhalation sortie des abîmes » et une puante haleine à l'orée de Laringues et Paringues.

Le gosier stimule donc l'idée de passage. La bouche est une voie de communication, une porte à battants ouverts. Le corps absorbe et restitue. Puisque la représentation anthropophagique de la bouche a brouillé les frontières du corps et du monde, c'est à un corps cosmique communiquant, tel qu'il a été célébré par Bakhtin, que l'on a affaire : un corps grotesque ouvert à tous les vents, dans lequel se tapissent des univers inconnus et mouvants («XXV royaumes habités»), « profondeurs fécondes et excroissances reproductrices » :

«De la sorte, tous les organes et lieux essentiels du corps grotesque, tous les éléments importants qui affectent sa vie sont développés et dépeints autour de l'image centrale de la bouche ouverte. Celle-ci est l'expression la plus éclatante du corps ouvert, non clos. » ${ }^{33}$

La bouche grotesque est organiquement associée à des phénomènes de déglutition, absorption, et éjection, depuis le haut vers le bas, du ventre, aux entrailles à l'accouchement, tout cela étant symptomatique d'un corps ouvert. L'aller-retour des ingestions et des excrétions, sera plus largement décrit au chapitre suivant où Pantagruel fait l'objet d'un lavage d'estomac, dans le dessein de rétablir la fonction communicante de son gosier. L'estomac, comme la bouche,

\footnotetext{
${ }^{32}$ Rigolot, 122.

${ }^{33}$ Bakhtin, 336.
} 
y sera dépeint à échelle grandiose et de façon cosmique, à la manière d'un enfer joyeux.

\section{Arrière goût}

Tel que le proposerait un récit de voyage, on attend maintenant de l'explorateur qu'il apporte une conclusion et une interprétation personnelle de ce qu'il a vu, entendu et appris. Cette conclusion semble s'esquisser à l'issu des six mois qu'Alcofribas a passé dans la bouche de Pantagruel, occupé pour moitié à dormir, voire à ronfler (encore mieux payé, aux dires de l'auteur). Elle aboutit à une première constatation, celle que l'ancien monde et le nouveau partagent les même affres, sont soumis aux mêmes démêlés entre les gens « de deçà et de delà » - on pense à la vraie querelle entre les Réformateurs et les Evangélistes. Ensuite, Alcofribas se hasarde à généraliser son expérience en proposant l'observation suivante :

«Là commençai penser qu'il est bien vrai ce que l'on dit, que la moitié du monde ne sait pas comment l'autre vit. » ${ }^{34}$

Encore que cette pensée, apparemment mûrement digérée, ne s'appuie pas sur son expérience personnelle mais, ironiquement, sur un «quand dira-t-on » qui dispense Alcofribas de penser par lui-même. Finalement, l'ultime enjeu de cette relation de voyage nous demeure caché, puisqu'il s'agit du livre sur l'Histoire des Guorgias et la seule mention de cet ouvrage évite au chroniqueur de nous en dire davantage.

Après la conclusion éludée, Alcofribas s'en retourne de cet Ailleurs si semblable à son $I c i$, et se retrouve face à face avec son hôte, Pantagruel. Le comique provient du fait que Pantagruel n'a rien senti de la présence de l'auteur dans sa gorge, en raison de l'infranchissable rapport d'échelle qui existe entre eux deux, entre microcosme et macrocosme : là encore, voici deux mondes qui ne se connaissent pas. La curiosité de Pantagruel à l'égard de ce petit être, sorti droit de sa gorge, est réelle, et au lieu de concerner l'exotisme du voyage, elle s'adresse seulement au fonctionnement du système digestif d'Alcofribas. Autrement dit, la curiosité de Pantagruel porte sur les bouches : «que beuvais-yu ?», « où chiaistu ? ». La réponse «En votre gorge... » superpose de façon ludique une expression toute faite (ce que l'on rétorquait à l'époque quand l'autre disait «merde ») à une réponse «en langue naturelle», signalant simplement le lieu géographique de l'expédition. Connaître l'autre, c'est donc, selon Pantagruel, connaître l'état de son organe d'ingestion/digestion. La topographie de la bouche fournit une carte d'identité qui permet d'évaluer la qualité de la langue-organe, qui «parle » pour la langue naturelle. Pantagruel, avant de prendre pour ami Panurge, s'était de même assuré qu'il parlait en sa langue naturelle, le français. Une analogie troublante est ici suggérée par Rabelais entre Alcofribas et Panurge. D'une part l'analogie concerne la châtellenie de Salmigondin (en référence, semble-t-il, à la ville de Saumur en Anjou, autre allusion au sel) que Pantagruel leur lègue successivement dans le récit: cette demeure reviendra en effet à Panurge, au Tiers Livre, quand

${ }^{34}$ Rabelais, II.XXVIII, 523. 
Alcofribas aura disparu, et lui sera attribué selon les mêmes formules. D'autre part la reconnaissance d'amitié entre les deux personnages est identique, immédiate et non raisonnée. Si Pantagruel partageait avec Panurge le même «jardin de France », la Touraine, avec Alcofribas, il partage cette fois les mêmes excréments et le même fonctionnement physiologique. Comme on est frères de sang dans la vie, on est frères de défécation au pays des Almyrodes.

A l'issu du voyage, que retiendra-t-on de cette mise en bouche ? Si l'intérieur semble refléter l'extérieur, avec l'indifférence partagée que les uns portent aux autres, Alcofribas en appelle à une reconnaissance réciproque. " Nouveau monde » pour Alcofribas, «terre neuve » pour le planteur de choux, c'est «bonnet blanc et blanc bonnet». L'essentiel est de reconnaître l'autre comme un semblable. Le gage spontané d'amitié de Pantagruel envers ses deux amis (ou deux marottes), répond à la même sagesse. Il y a réciprocité, et non communication à sens unique. Contrairement à la bouche de l'enfer, sans retour, on ressort de cette bouche grotesque avec gaieté, le rire permettant de mieux retenir la leçon de sagesse. Car la multiplicité des expériences, la variété des niveaux de langue, la charité envers les uns les autres, font désormais parti du bagage d'Alcofribas, le découvreur. 


\section{BIBLIOGRAPHIE}

AUERBACH Erich, Mimesis; the Representation of Reality in Western Literature. Princeton, Princeton University Press, 1953.

BAHTIN Mihail Mihailovič, Andrée RoBEL. L'Euvre De François Rabelais Et La Culture Populaire Au Moyen Âge Et Sous La Renaissance Traduit Du Russe Par André Robel. Paris, Gallimard, 1970.

BERTRAND Dominique (éd.) L'horrible Et Le Risible. Vol. 14, Humoresques, CORHUM, Juin 2001.

CAMILLE Michael. Image on the Edge : The Margins of Medieval Art, Essays in Art and Culture. Cambridge, Mass., Harvard University Press, 1992.

Chastel André. La Grottesque. Paris, Le Promeneur, 1988.

DE GRÈVE Marcel, Claude DE GRÈVE, Jean CÉARD, La Réception De Rabelais En Europe Du XVIe Au XVIIIe Siècle, Études réunies par Claude De Grève et Jean Céard, Études et essais sur la Renaissance. Paris, H. Champion, 2009.

DEMERSON Guy, François RABElaIs, L' Esthétique De Rabelais, Esthétique, Paris, Sedes, 1996.

ERASME. La Langue. Vol. 44, Histoire et Société, Labor et Fides, 2002.

ERASMUS Desiderius, William BARKER. The Adages of Erasmus. Toronto, University of Toronto Press, 2001.

FouCAUlt Michel, Histoire De La Folie À L'âge Classique, Collection Tel. Paris, Gallimard, 1992.

GuÉDron Martial. L' Art De La Grimace Cinq Siècles D'excès De Visage. Paris, Hazan, 2011.

JEANNERET Michel, Le Défi Des Signes Rabelais Et La Crise De L'interprétation À La Renaissance, Collection L'atelier de la Renaissance, Orléans, Paradigme, 1994.

KAISER Walter Jacob. Praisers of Folly: Erasmus, Rabelais, Shakespeare, Harvard Studies in Comparative Literature, Cambridge, Harvard University Press, 1963.

LeVER Maurice, Le Sceptre Et La Marotte Histoire Des Fous De Cour, Paris, Fayard, 2000.

PINSON Yona, The Fools' Journey: A Myth of Obsession in Northern Renaissance Art, Turnhout, Brepols, 2008.

RABElais François, Les Cinq Livres. Jean CÉARD, Gérard DEFAUX, Michel SimONIN (éds.), La Pochothèque. Paris, Le Livre de Poche, 1994.

Rabelais François, Michel JeanNeret, Frédéric Elsig, François DesPreZ, Les Songes Drolatiques De Pantagruel Postface De Frédéric Elsig, Titre Courant. Genève, Droz, 2005.

RigOLOT François, Les Langages De Rabelais, Études Rabelaisiennes. Genève, Droz, 1972.

Sullivan Margaret A., Pieter Bruegel, Bruegel and the Creative Process, 15591563. Farnham, Surrey, England ; Burlington, VT: Ashgate, 2010.

WIND Barry. A Foul and Pestilent Congregation': Images of "Freaks 》 in Baroque Art. Aldershot, England ; Brookfield, Vt., USA: Ashgate, 1998.

ZORACH Rebecca. Blood, Milk, Ink, Gold: Abundance and Excess in the French Renaissance, Chicago, University of Chicago Press, 2005. 


\section{ILLUSTRATIONS}

Fig. 1 - Annibale Carrache : Le mangeur de pois, 1580-90. Huile sur toile, Galerie Colonna, Rome

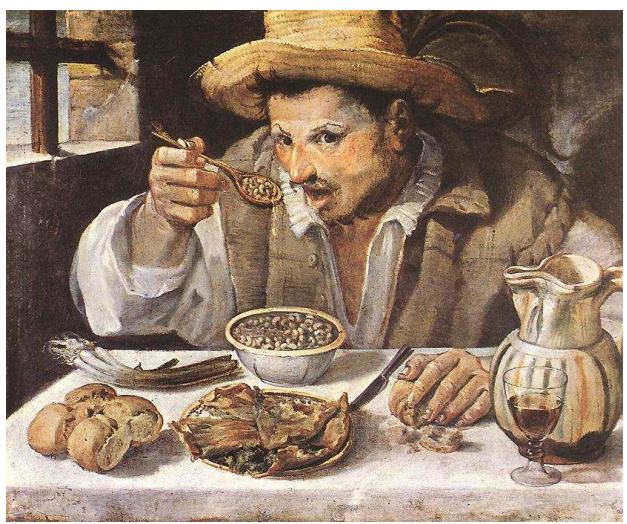

Fig. 3 - Le Maître de Catherine de Clèves, La Gueule de l'Enfer, vers 1440, New York, Pierpont, Morgan Library

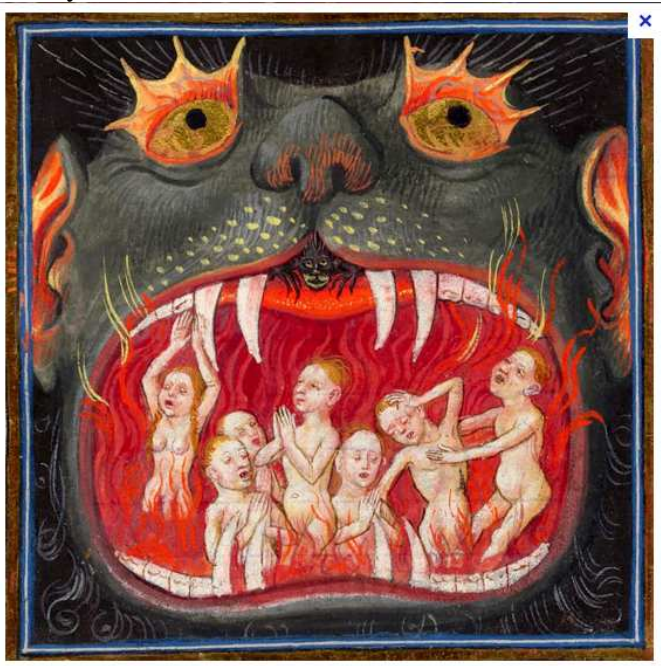

Fig. 2 - Marbre grec, $\mathrm{II}^{\mathrm{e}}$ siècle avant J.C.

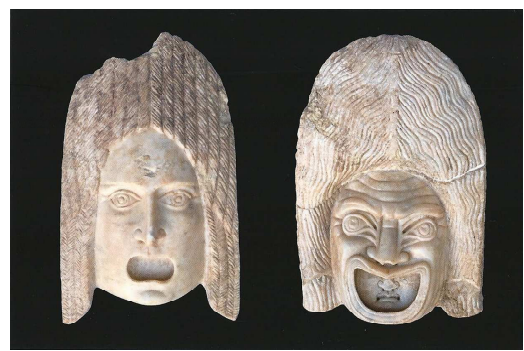

Fig.4 - Lucifer, Codex Altonensis, XIV ${ }^{\mathrm{e}}$ siècle, folio $48 \mathrm{r}$

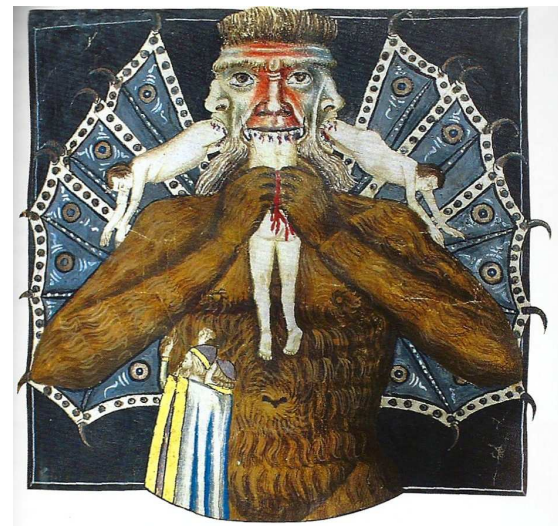


Fig.5 - Jérôme Bosch, Tryptique du Jardin des Délices, 1503. Détail de L’Enfer, Musée du Prado, Madrid

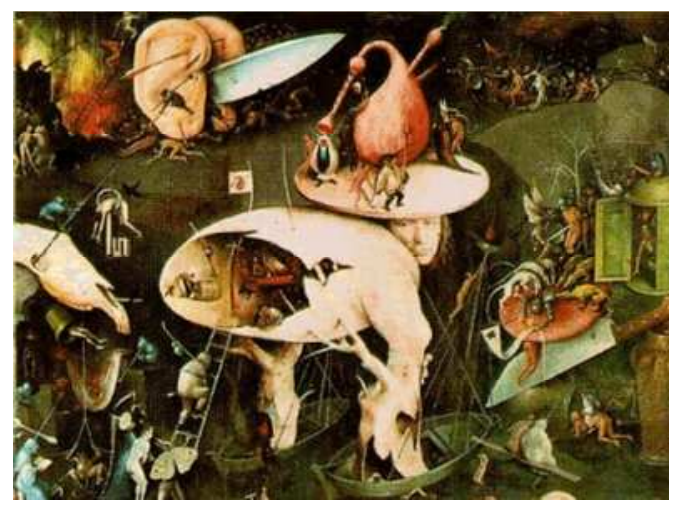

Fig. 7 - Face-pulling heads. Reims Cathedral.
Fig. 6 - Lettrines

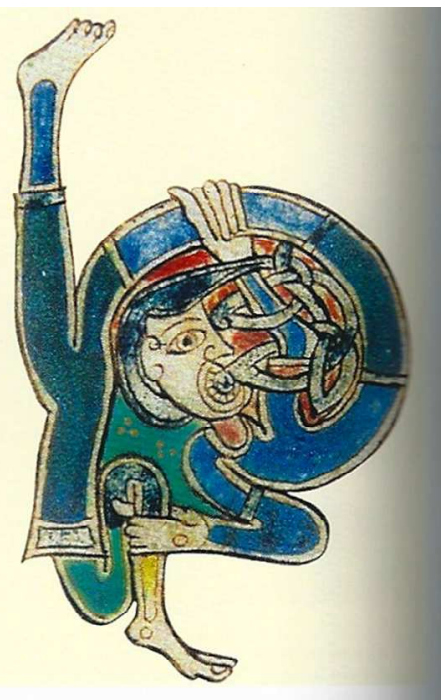

Fig. 8 - Gargouille,

Hotel de Sade, Avignon

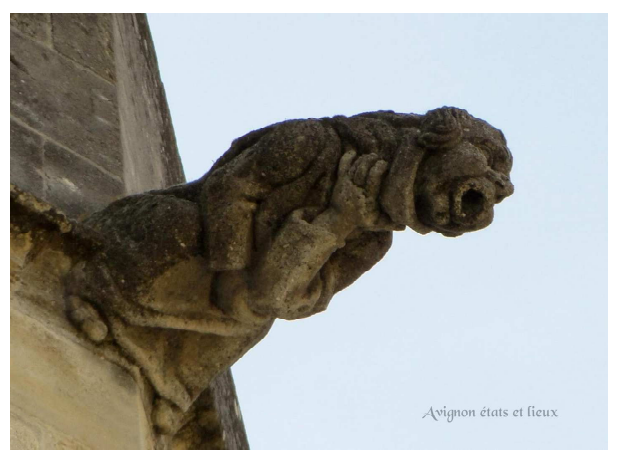


Fig. 9 - Shitting for one's lady, livre d'heures, Trinity College, Cambridge

Fig. 11 - Anonyme, Bouffon, XVIe siècle Huile sur bois, Chambéry, musée des Beaux-Arts

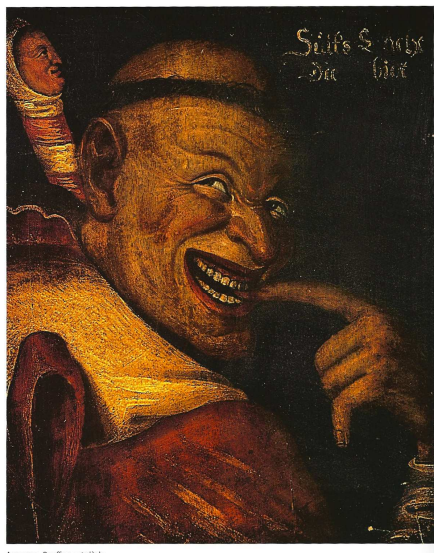

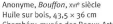

Fig. 13 - anonyme, Le monde est fou, Gravure, Anvers

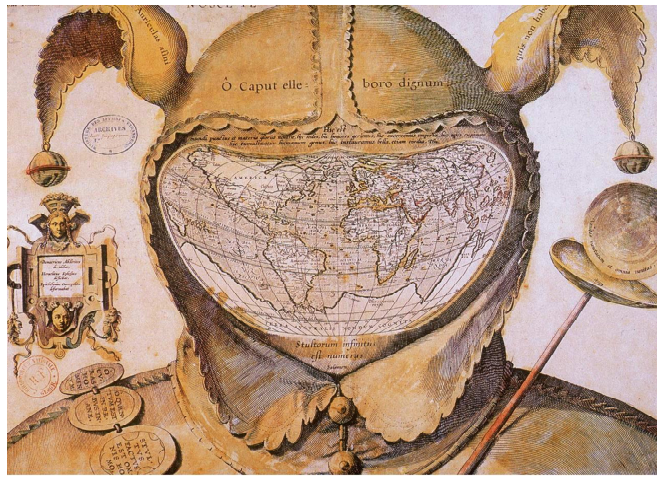

Fig. 10 - Jérôme Bosch, Nef des Fols, vers 1500. Huile sur panneau, Musée du Louvre, Paris

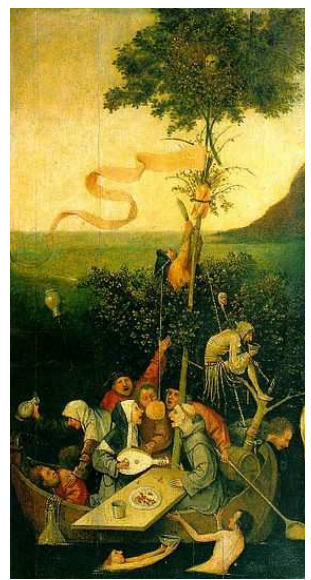

Fig. 12 - Drawing by Holbein.

Marginal illustration for Erasmus' Praise of Folly.

\section{Iuuentus}

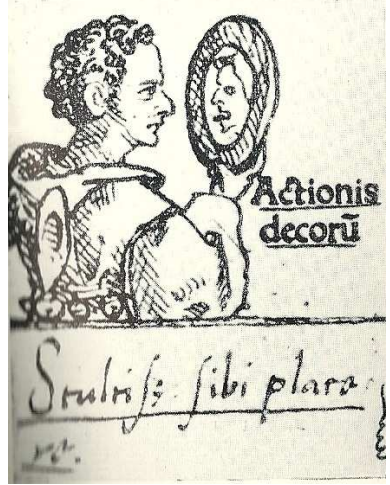

Fig. 14 - Pieter Bruegel l'Ancien,

Les gros poissons mangent les petits, 1556

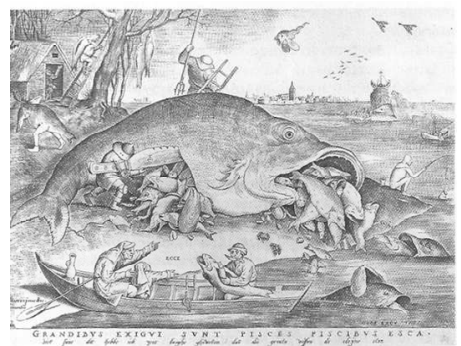


Fig. 15 - Attribué à Pieter Brueghel le jeune, Le Bâilleur, 1616.

Huile sur bois, Bruxelles, Musée Royaux des BeauxArts

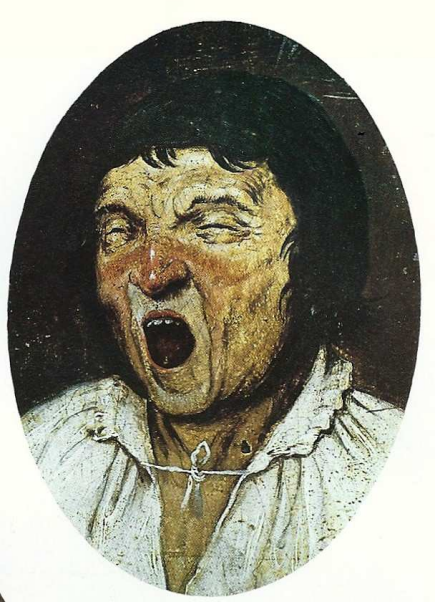

